\title{
CHARACTERISTIC ROOTS AND FIELD OF VALUES OF A MATRIX
}

\author{
W. V. PARKER
}

1. Introduction. Let $A=\left(a_{i j}\right)$ be a square matrix of order $n$ whose elements are in the field of complex numbers. The complex number $\lambda$ is a characteristic root of the matrix $A$ if the determinant of the matrix $\lambda I-A$ is zero. It follows that $\lambda$ is a characteristic root of $A$ if, and only if, there exists a vector $x$ such that $x x^{*}=1$ and

$$
A x^{*}=\lambda x^{*}
$$

where * is used to denote transposed conjugate. By taking transposed conjugates on both sides in (1) we obtain

$$
x A^{*}=\bar{\lambda} x \text {. }
$$

From (1) it follows that $\lambda=x A x^{*}$. The set of all complex numbers $z A z^{*}$ where $z z^{*}=1$ is called the field of values $[25]^{1}$ of the matrix $A$. It follows that the characteristic roots of $A$ belong to the field of values of $A$.

Beginning with Bendixson [3] in 1900, many writers have obtained limits for the characteristic roots of a matrix. In many cases these were actually limits for the field of values of the matrix [14]. In an address delivered before the Mathematical Association of America in 1938, Browne [10] gave a summary of these results up to that time. It is the purpose here to discuss some of the results obtained since the time of Browne's paper.

2. Some well known results. If $x$ and $y$ are two vectors such that $x x^{*}=y y^{*}=1$ and $X$ and $Y$ are unitary matrices with leading vectors $x$ and $y$ respectively, then $x X^{*}=y Y^{*}$ and hence $y=x X^{*} Y$, or $y=x U$ where $U$ is a unitary matrix. Also, if $x x^{*}=1$ and $y=x U$ where $U$ is a unitary matrix, then $y y^{*}=x U U^{*} x^{*}=x x^{*}=1$. It follows, therefore, that $A$ and $U A U^{*}$ have the same field of values and the same characteristic roots for every unitary matrix $U$. It may be readily shown [21] that the field of values of $A$ is identical with the set of all diagonal elements of the matrices $U A U^{*}$ where $U U^{*}=I$. If $A$ is Hermitian there exists a unitary matrix $U$ such that $U A U^{*}=$ diag. $\left\{\lambda_{1}, \lambda_{2}, \cdots, \lambda_{n}\right\}$

An addressed delivered before the Oak Ridge meeting of the Society, April 21, 1950, by invitation of the Committee to Select Hour Speakers for Southeastern Sectional Meetings; received by the editors June 28, 1950.

1 Numbers in brackets refer to the bibliography at the end of the paper. 
where $\lambda_{1} \leqq \lambda_{2} \leqq \cdots \leqq \lambda_{n}$ are the (real) characteristic roots of $A$. It follows immediately that the field of values of an Hermitian matrix is the shortest segment of the real axis containing all the characteristic roots of $A$.

From (1) and (2) the following relations are obtained

$$
\begin{gathered}
\lambda=x A x^{*}, \quad \bar{\lambda}=x A^{*} x^{*}, \quad \bar{\lambda} \lambda=x A^{*} A x^{*}, \\
\frac{\lambda+\bar{\lambda}}{2}=x\left(\frac{A+A^{*}}{2}\right) x^{*}, \quad \frac{\lambda-\bar{\lambda}}{2 i}=x\left(\frac{A-A^{*}}{2 i}\right) x^{*} .
\end{gathered}
$$

From the first three of these relations we obtain two well known theorems. If $A=A^{*}$, then $\lambda=\bar{\lambda}$ and hence the characteristic roots of an Hermitian matrix are all real. If $A^{*} A=I$, then $\bar{\lambda} \lambda=1$, and hence the characteristic roots of a unitary matrix are all in absolute value one. Again from the third of these relations we obtain at once Browne's theorem [8] that $|\lambda|$ is bounded by the least and greatest of the characteristic roots of the positive semi-definite Hermitian matrix $B$ defined by $B^{2}=A^{*} A$. It may also be shown [20] that for a given $B$ there exists a matrix $A$ which has as a characteristic root any number in this region. From the last two relations in (3) it follows that the characteristic roots of $A$ lie in a rectangular region of the complex plane determined by the characteristic roots of $\left(A+A^{*}\right) / 2$ and $\left(A-A^{*}\right) / 2$ [16]. In fact the entire field of values of $A$ lies in this rectangle.

3. Limits for the characteristic roots of a matrix. In 1930 Browne [9] obtained a limit for the characteristic roots of the matrix $A$ in terms of the sums of the absolute values of the elements in the rows and the columns of $A$. Write

$$
\begin{gathered}
R_{i}=\sum_{i}\left|a_{i j}\right|, \quad T_{i}=\sum_{i}\left|a_{i j}\right|, \quad 2 S_{i}=R_{i}+T_{i}, \\
R_{i}^{\prime}=R_{i}-\left|a_{i i}\right|, \quad T_{i}^{\prime}=T_{i}-\left|a_{j i}\right|
\end{gathered}
$$

and let $R, T$, and $S$ denote the maximum $R_{i}, T_{i}$, and $S_{i}$, respectively. Browne showed that $|\lambda| \leqq(R+T) / 2$. This result was improved by this author in 1937 [18] when it was shown that $|\lambda| \leqq S \leqq(R+T) / 2$. Another improvement of Browne's result was given by Farnell in 1944 when he showed that $|\lambda| \leqq(R T)^{1 / 2} \leqq(R+T) / 2$. In 1945 Barankin [1] further improved Farnell's result by showing $|\lambda| \leqq \max \left(R_{i} T_{i}\right)^{1 / 2}$.

The first two of these are actually limits for the field of values of $A$. Suppose that $\mu=x A x^{*}$ is any number in the field of values of $A$. Write $x=\left(x_{1}, x_{2}, \cdots, x_{n}\right)$, then $\mu=\sum_{i, j} a_{i j} x_{i} \bar{x}_{j}$. If $\left|x_{i}\right|=\xi_{i}$ it follows that 


$$
\begin{aligned}
|\mu| & \leqq \sum_{i, i}\left|a_{i j}\right| \xi_{i} \xi_{j} \leqq \frac{1}{2} \sum_{i, j}\left|a_{i j}\right|\left(\xi_{i}^{2}+\xi_{i}^{2}\right) \\
& =\frac{1}{2} \sum_{i} R_{i} \xi_{i}^{2}+\frac{1}{2} \sum_{i} T_{i} \xi_{i}^{2}=\sum_{i} S_{i} \xi_{i}^{2} \leqq S \sum_{i} \xi_{i}^{2}=S .
\end{aligned}
$$

Relation (1) may be written as a system of linear equations in the form

$$
\lambda \bar{x}_{i}=\sum_{i} a_{i j} \bar{x}_{i} \quad(i=1,2, \cdots, n) .
$$

If $\xi_{k}$ is the greatest of the $\xi_{i}$, then since $\lambda \bar{x}_{k}=\sum_{j} a_{k j} \bar{x}_{j}$

$$
\left|\lambda \bar{x}_{k}\right|=|\lambda| \xi_{k} \leqq \sum_{j}\left|a_{k j}\right| \xi_{i} \leqq R_{k} \xi_{k}
$$

and hence $|\lambda| \leqq R_{k}$. In a similar manner $|\lambda| \leqq T_{m}$ for some $j=m$. This establishes the following theorem.

THEOREM 1. If $\lambda$ is a characteristic root of the matrix $A$ and $R$ is the greatest sum obtained for the absolute values of the elements of a row and $T$ is the greatest sum obtained for the absolute values of the elements of a column, then $|\lambda| \leqq \min (R, T)$.

This theorem was proved by the author in 1943. It was subsequently given by Barankin [1] and later again by Brauer [4]. In an added note Brauer pointed out that the theorem was proved by Perron in the second edition of his Algebra in 1933 and also by Specht in 1938. In this same paper Brauer obtained a better limit given by the following theorem.

THEOREM 2. Let $M_{r}$ be the maximum of the sums of the absolute values of the elements in each row of the matrix $A^{2^{r}}$. Then each characteristic root $\lambda$ of $A$ satisfies $|\lambda| \leqq\left(M_{r}\right)^{1 / 2^{r}}$.

From (5) it follows that

$$
\left(\lambda-a_{k k}\right) \bar{x}_{k}=\sum_{j \neq k} a_{k j} \bar{x}_{j}
$$

so that

$$
\left|\lambda-a_{k k}\right| \xi_{k} \leqq \sum_{j \neq k}\left|a_{k j}\right| \xi_{j} \leqq \xi_{k} \sum_{j \neq k}\left|a_{k j}\right|=R_{k}^{\prime} \xi_{k}
$$

and hence

$$
\left|\lambda-a_{k k}\right| \leqq \sum_{j \neq k}\left|a_{k j}\right|=R_{k}-\left|a_{k k}\right|=R_{k}^{\prime}
$$


In a similar fashion

$$
\left|\lambda-a_{m m}\right| \leqq T_{m}-\left|a_{m m}\right|=T_{m}^{\prime} .
$$

This establishes the following theorem.

TheOREM 3 (BRAUER [4]). Let $A=\left(a_{i j}\right)$ be an arbitrary matrix and $R_{k}^{\prime}$ the sum of the absolute values of the non-diagonal elements of the kth row and $T_{m}^{\prime}$ the sum of the absolute values of the non-diagonal elements of the mth column of $A$. Each characteristic root of $A$ lies in at least one of the circles $\left|z-a_{k k}\right| \leqq R_{k}^{\prime}$ and in at least one of the circles $\left|z-a_{m m}\right|$ $\leqq T_{m}^{\prime}$.

The equations (5) may also be written as

$$
\left(\lambda-a_{i i}\right) \bar{x}_{i}=\sum_{j \neq i} a_{i j} \bar{x}_{j} \quad(i=1,2, \cdots, n) .
$$

If $\xi_{k} \geqq \xi_{l} \geqq \xi_{j}, j \neq k, l$, then from (6)

$$
\left|\lambda-a_{k k}\right| \xi_{k} \leqq R_{k}^{\prime} \xi_{l} \text { and }\left|\lambda-a_{l l}\right| \xi_{l} \leqq R_{l}^{\prime} \xi_{k} .
$$

It is assumed now that the characteristic root $\lambda$ is not the diagonal element $a_{k k}$, so that $\xi_{l} \neq 0$. Then since

$$
\left|\lambda-a_{l k}\right| \cdot\left|\lambda-a_{l l}\right| \xi_{k} \xi_{l} \leqq R_{k}^{\prime} R_{l}^{\prime} \xi_{k} \xi_{l}
$$

it follows that

$$
\left|\lambda-a_{k k}\right| \cdot\left|\lambda-a_{l l}\right| \leqq R_{k}^{\prime} R_{l}^{\prime}
$$

The inequality is obviously true of $\lambda=a_{k k}$. In a similar manner

$$
\left|\lambda-a_{m m}\right| \cdot\left|\lambda-a_{q q}\right| \leqq T_{m}^{\prime} T_{q}^{\prime}
$$

for some $m$ and $q$. This establishes the following theorem.

TheOREM 4 (BRAUER II [5]). Each characteristic root $\lambda$ of the matrix $A$ lies in at least one of the $n(n-1) / 2$ ovals of Cassini

$$
\left|z-a_{k k}\right| \cdot\left|z-a_{l l}\right| \leqq R_{k}^{\prime} R_{l}^{\prime}
$$

and in at least one of the ovals

$$
\left|z-a_{k k}\right| \cdot\left|z-a_{l l}\right| \leqq T_{k}^{\prime} T_{l}^{\prime}
$$

In his third paper Brauer [6] shows how the above theorems may be applied to specially selected polynomials in $A$ to obtain better limits for the characteristic roots. He proved the following theorem. 
Theorem 5 (Brauer III [6]). Let $A=\left(a_{i j}\right)$ be a square matrix of order $n$ and $f_{1}(y), f_{2}(y), \cdots, f_{n}(y)$ be arbitrary polynomials. Denote the elements of the matrix $f_{r}(A)$ by $a_{i j}^{\left(f_{r}\right)}$ and set

$$
\sum_{j=1, j \neq i}^{n}\left|a_{i j}^{\left(f_{r}\right)}\right|=P_{i}^{\left(f_{r}\right)} \quad(i, r=1,2, \cdots, n)
$$

Each characteristic root $\lambda$ of $A$ satisfies at least one of the $n$ inequalities

$$
\left|f_{s}(\lambda)-a_{s s}^{\left(f_{s}\right)}\right| \leqq P_{s}^{\left(f_{s}\right)} \quad(s=1,2, \cdots, n) .
$$

and at least one of the $n(n-1) / 2$ inequalities

$$
\begin{aligned}
\left|f_{s}(\lambda)-a_{s s}^{\left(f_{s}\right)}\right| \cdot\left|f_{t}(\lambda)-a_{t t}^{\left(f_{t}\right)}\right| & \leqq P_{s}^{\left(f_{s}\right)} P_{t}^{\left(f_{t}\right)} \\
& (s, t=1,2, \cdots, n ; s \neq t) .
\end{aligned}
$$

4. Limits for the field of values of a matrix. As pointed out above, several of the earlier theorems actually gave upper bounds for the field of values of $A$. Farnell [14] gives two limits for the field of values of $A$. Let $d_{r s}=\left(\left|a_{r s}\right|+\left|a_{s r}\right|\right) / 2$, and let $S_{r}=\sum_{s} d_{r s .}^{2}$. Then if $\lambda$ lies in the field of values of $A,|\lambda| \leqq \max \left(S_{r}\right)$ and $|\lambda| \leqq\left(\sum_{r, s} d_{r s}^{2}\right)^{1 / 2}$.

In most instances the above bounds are obtained in terms of the distance from zero. However zero may lie entirely outside the field of values of $A$. Since the field of values of $A$ is convex and contains all the elements of the diagonal of $A$, it also contains the centroid of these elements. That is, $c=(1 / n) \sum_{i} a_{i i}$ belongs to the field of values of $A$. Since the trace, $T(A)=\sum_{i} a_{i i}$, is invariant under unitary transformations, the same $c$ is obtained for every $U A U^{*}$ where $U$ is unitary. In fact it is possible to select $U$ so that every diagonal element of $U A U^{*}$ will be $c[\mathbf{2 2}]$.

If $B=A-c I$, the field of values of $B$ is obtained by shifting the field of values of $A$ the distance $|c|$ in the direction of $-c$. Applying the above theorems to $B$ now gives bounds for the characteristic roots of $A$ and the field values of $A$ in terms of the distance from $c$. This was used by the author [22] to obtain a theorem on the spread of the characteristic roots of an Hermitian matrix.

THEOREM 6. If $A=\left(a_{i j}\right)$ is an Hermiitan matrix with characteristic roots $\lambda_{1} \leqq \lambda_{2} \leqq \cdots \leqq \lambda_{n}$, then

$$
\left(\lambda_{n}-\lambda_{1}\right) / 2 \geqq \max \left|a_{i j}\right|, \quad i \neq j .
$$

\section{BIBLIOGRAPHY}

1. E. W. Barankin, Bounds for the characteristic roots of a matrix, Bull. Amer. Math. Soc. vol. 51 (1945) pp. 767-770. 
2. - Bounds on characteristic values, Bull. Amer. Math. Soc. vol. 54 (1948) pp. 728-735.

3. I. Bendixson, Sur les racines d'une équation fondamentale, Acta Math. vol. 25 (1902) pp. 359-365.

4. Alfred Brauer, Limits for the characteristic roots of a matrix, Duke Math. J. vol. 13 (1946) pp. 387-395.

5. - Limits for the characteristic roots of a matrix II, Duke Math. J. vol. 14 (1947) pp. 21-26.

6. - Limits for the characteristic roots of a matrix III, Duke Math. J. vol. 15 (1948) pp. 871-877.

7. T. J. I'A. Bromwich, On the roots of the characteristic equation of a linear substitution, Acta Math. vol. 30 (1906) pp. 295-304.

8. E. T. Browne, The characteristic equation of a matrix, Bull. Amer. Math. Soc. vol. 34 (1928) pp. 363-368.

9. - The characteristic roots of a matrix, Bull. Amer. Math. Soc. vol. 36 (1930) pp. 705-710.

10. - Limits to the characteristic roots of a matrix, Amer. Math. Monthly vol. 46 (1939) pp. 252-265.

11. J. C. Currie, The field of values of a matrix, Dissertation, Louisiana State University, 1948.

12. - Cassini ovals associated with a second order matrix, Amer. Math. Monthly vol. 55 (1948) pp. 487-489.

13. A. B. Farnell, Limits for the characteristic roots of a matrix, Bull. Amer. Math. Soc. vol. 50 (1944) pp. 789-794.

14. - Limits for the field of values of a matrix, Amer. Math. Monthly vol. 52 (1945) pp. 488-493.

15. F. Hausdorff, Der Wertvorrat einer Bilinearform, Math. Zeit. vol. 3 (1919) pp. 314-316.

16. A. Hirsch, Sur les racines d'une équation fondamentale, Acta Math. vol. 25 (1902) pp. 367-370.

17. F. D. Murnaghan, On the field of values of a square matrix, Proc. Nat. Acad. Sci. U.S.A. vol. 18 (1932) pp. 246-248.

18. W. V. Parker, The characteristic roots of a matrix, Duke Math. J. vol. 3 (1937) pp. $484-487$.

19. - Limits to the characteristic roots of a matrix, Duke Math. J. vol. 10 (1943) pp. 479-482.

20. — The characteristic roots of matrices, Duke Math. J. vol. 12 (1945) pp. $519-526$.

21. - Characteristic roots and the field of values of a matrix, Duke Math. J. vol. 15 (1948) pp. 439-442.

22. - Sets of complex numbers associated with a matrix, Duke Math. J. vol. 15 (1948) pp. 711-715.

23. O. Taussky, Bounds for characteristic roots of matrices, Duke Math. J. vol. 15 (1948) pp. 1043-1044.

24. - $-A$ recurring theorem on determinants, Amer. Math. Monthly vol. 56 (1949) pp. 672-676.

25. O. Toeplitz, Das algebraische Analogon zu einem Satze von Fejer, Math. Zeit. vol. 2 (1918) pp. 187-197.

Alabama Polytechnic Institute 